\title{
A sound-based measurement of sway angle for anti-sway control of overhead crane
}

\author{
Miki Matsunaga \\ Graduate School of Engineering, Hiroshima University, 1-4-1, Kagamiyama, \\ Higashi-Hiroshima city, Hiroshima, Japan \\ Masayoshi Nakamoto \\ Graduate School of Engineering, Hiroshima University, 1-4-1, Kagamiyama, \\ Higashi-Hiroshima city, Hiroshima, Japan \\ Toru Yamamoto \\ Graduate School of Engineering, Hiroshima University, 1-4-1, Kagamiyama, \\ Higashi-Hiroshima city, Hiroshima, Japan \\ E-mail: matsunaga-miki@hiroshima-u.ac.jp,msy@hiroshima-u.ac.jp, \\ yama@hiroshima-u.ac.jp
}

\begin{abstract}
For anti-swing control of overhead crane, a deflection angle must be estimated. However, it is difficult to estimate deflection angles with a contact sensor such as rotary encoder. Therefore, we show a non-contact measurement method for the deflection angles by using two microphones. This method is based on the sound source location in a human with two ears. The method employs a time delay of arrival (TDOA) of acoustic signals which are picked up by the two microphones. Also, we show an algorithm to obtain the angle from the TDOA by using the Newton's method. Finally, we show experimental results to demonstrate the effectiveness of the proposed method.
\end{abstract}

Keywords: Overhead crane, microphones, correlation function, Newton's method

\section{Introduction}

Overhead cranes are well-used for transportation of load in factory and dock. For safety and efficiency transportation with the overhead cranes, the anti-sway control $^{1-5}$ has been received remarkable attention.

Since the dynamics of the sway angle of the crane can be described by the state-space equations, the sway can be controlled. However, the sway angle should be obtained for anti-sway control. It is known that the measurement of the sway angle is very difficult with contact sensor due to the hardware constraint.

From the background above, we focus on the sound signal $^{6}$ for the anti-sway control of overhead cranes. This scheme is inspired by the human hearing mechanism with two ears. Our measurement scheme is to obtain the sway angle without attached angle sensor. Here, we assume the payload includes a sound source such as friction sound, squeak noise or active noise. With two microphones, we detect the sound (acoustic) signal generated in the payload. That is, we use the microphones for contact-less sensing of the angle. Next, we estimate the time delay of arrival (TDOA) between the acoustic signals obtained from the two microphones where the TDOA can be computed from the correlation function. Also, we transform the TDOA to the sway angle by using the Newton's method.

In the experiment, we treat the case when the payload is swaying and the position of trolley is changed. From 
experimental results, we show the proposed method can follow the sway angle even when the payload is moving.

\section{Anti-sway Control of Overhead Crane}

In the crane, a road is moved by motor driven trolley. Table I shows the parameters of the overhead crane.

Let $l_{w}$ be the distance from fulcrum of the trolley to the payload (sound source). $x(0 \leqq x \leqq X)$ is the position of the trolley where $\mathrm{x}=0$ is the left end and $x=X$ is the right end. That is, the distance between the Microphone 1 and the trolley is $x$ (variable). Also, $\theta$ is the angle of the line (sway angle). Assume $T$ is kinetic energy and $U$ is potential energy. Then, we have

$$
\begin{aligned}
T & =\frac{1}{2} M \dot{x}^{2}+\frac{1}{2} m v^{2} \\
U & =m g l_{w}(1-\cos \theta)
\end{aligned}
$$

Also, define $L=T-U$ is the Lagrangian. Now, let the state vector be

$$
x(t)=\left[\begin{array}{llll}
x-x_{d} & \theta & \dot{x} & \dot{\theta}
\end{array}\right]^{\mathrm{T}},
$$

where $x_{d}$ is the desired position, and its time derivation be

$$
\dot{x}(t)=\left[\begin{array}{llll}
\dot{x} & \dot{\theta} & \ddot{x} & \ddot{\theta}
\end{array}\right]^{\mathrm{T}}
$$

Based on the Euler-Lagrange equation, the dynamics of the crane can be written in a state space from:

$$
\begin{aligned}
& \dot{x}(t)=\left[\begin{array}{cccc}
0 & 0 & 1 & 0 \\
0 & 0 & 0 & 1 \\
0 & \frac{m g}{M} & 0 & 0 \\
0 & -\frac{(M+m) g}{M l_{w}} & 0 & 0
\end{array}\right] x(t) \\
& +\left[\begin{array}{c}
0 \\
0 \\
\frac{1}{M} \\
-\frac{1}{m l_{w}}
\end{array}\right] u(t) \\
& y(t)=\left[\begin{array}{llll}
1 & 0 & 0 & 0 \\
0 & 1 & 0 & 0
\end{array}\right] x(t)
\end{aligned}
$$

where $u(t)$ is an input signal, $y(t)$ is an output signal. Based on the state-space model above, $x$ and $\theta$ can be controlled using a state feedback. To achieve the control, the angle $\theta$ must be estimated.

Table 1. Parameters of the overhead crane

\begin{tabular}{|l|c|}
\hline weight of trolley & $M$ \\
\hline weight of payload & $m$ \\
\hline velocity of payload & $v$ \\
\hline external force & $f$ \\
\hline acceleration of gravity & $g$ \\
\hline
\end{tabular}

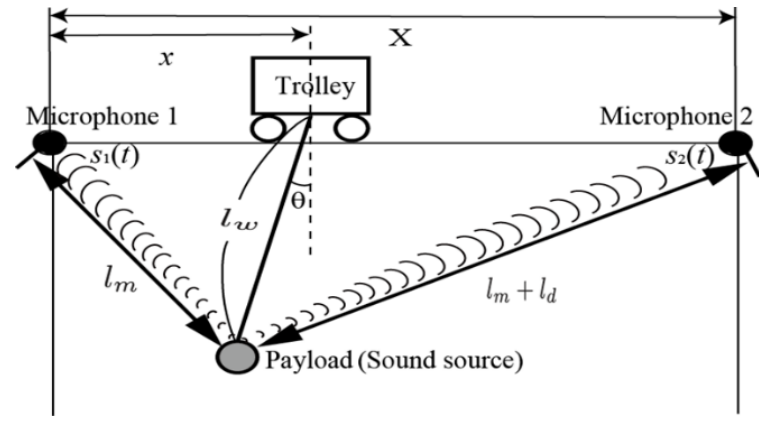

Fig. 1. Overhead crane with two microphones (Microphone 1 and Microphone 2) and sound source

\section{Sound-based Measurement of the Sway Angle}

\subsection{Estimate of the TDOA}

Fig. 1 shows the overhead crane system with two microphones (Microphone 1 and Microphone 2). With two microphones, we observe the sound signal originated from the sound source in the payload. Since the microphones have a directivity, the sensitivity of microphone is very low to the sound signal which is coming behind the microphones. Now, let $s_{1}(t)$ and $s_{2}(t)$ be the sound signal observed in the Microphone 1 and the Microphone 2, respectively. The distance between the two microphones is $\mathrm{X}$ since the microphone is located on the end of trolley rail. Further, let the distance between the Microphone 1 and the sound source be $l_{m}+l_{d}$ and the distance between the Microphone 2 and the sound source be $l_{m}$. Thus, $l_{d}$ is the difference of the distances. Assume that $x, X, l_{w}$, are known. Now, the purpose is to estimate $l_{d}$ based on the TDOA.

Using the two acoustic signals, we compute a correlation coefficients of $s_{1}(t+\tau) s_{2}(t)$ as follows. 


$$
\Phi(\tau)=\sum_{t=0}^{T_{0}} s_{1}(t+\tau) \cdot s_{2}(t)
$$

where $T_{0}$ the number of sampled point.

Let $\tau^{\prime}$ be the sampled point which maximizes (1).

Now, the TDOA between the Microphone 1 and the

Microphone 2 is $\tau^{\prime} T_{S}$ where $T_{s}$ is the sampling period.

If follows that

$$
l_{d}=\tau^{\prime} T_{s} v_{a}
$$

where $v_{a}$ is the velocity of sound.

\subsection{Conversion to angle $\theta$}

We introduce how to obtain $\theta$ with $l_{d}$. Using the law of cosines in Fig. 1, we have simultaneous equations as:

$$
\begin{gathered}
l_{m}=\sqrt{x^{2}+l_{w}{ }^{2}-2 x l_{w} \cos \left(\frac{\pi}{2}-\theta\right)} \\
l_{m}+l_{d}=\sqrt{(X-x)^{2}+l_{w}{ }^{2}-2(X-x) l_{w} \cos \left(\frac{\pi}{2}+\theta\right)}
\end{gathered}
$$

Now, eliminating $l_{m}$ in (3) and (4), we have a function below.

$$
\begin{aligned}
& f\left(\theta, l_{d}\right)=\left\{x^{2}+l_{w}{ }^{2}-2 x l_{w} \cos \left(\frac{\pi}{2}-\theta\right)\right\}^{\frac{1}{2}}+l_{d} \\
& -\left\{(X-x)^{2}+l_{w}{ }^{2}-2(X-x) l_{w} \cos \left(\frac{\pi}{2}+\theta\right)\right\}^{\frac{1}{2}}
\end{aligned}
$$

Hence, we can obtain $\theta$ corresponds $l_{d}$ by computing the solution which satisfies

$$
f\left(\theta, l_{d}\right)=0, \theta \in\left(-\frac{\pi}{2}, \frac{\pi}{2}\right)
$$

It should be noted that since $l_{m}$ can also be obtained from (3) or (4), sensor delay can also be estimated by the velocity of sound $v_{a}$.

Here, let us show how to compute the solution of (6).

Now, we employ the Newton's method to solve the solution of (6). The Newton's method uses the iterative equation as

$$
\theta_{n+1}=\theta_{n}-\frac{f\left(\theta_{n}, l_{d}\right)}{\left\{\frac{\partial f\left(\theta_{n}, l_{d}\right)}{\partial \theta}\right\}}
$$

\section{Experiment}

Fig. 2 shows the experimental equipment. We use a Gaussian random signal with $0 \sim 1000[\mathrm{~Hz}]$ as the sound source. The signal is generated by an electronic device. The sampling frequency is $14700[\mathrm{~Hz}]$. The correlation is calculated with data of samples corresponding to the position $x$. We consider the case when the payload is swaying (i.e. the sway angle is moving). Fig. 3 shows the result with $x=20$ where the vertical axis is the sway angle [deg.]. From Fig. 3, we can see that the payload is swaying around 0 [deg.]. In the same way, we show the results the position $x$ is changed as $x=30, x=40$, and $x=50$. Similarly, Figs. $4-6$ show the result with $x=30$, $x=40$, and $x=50$, respectively.

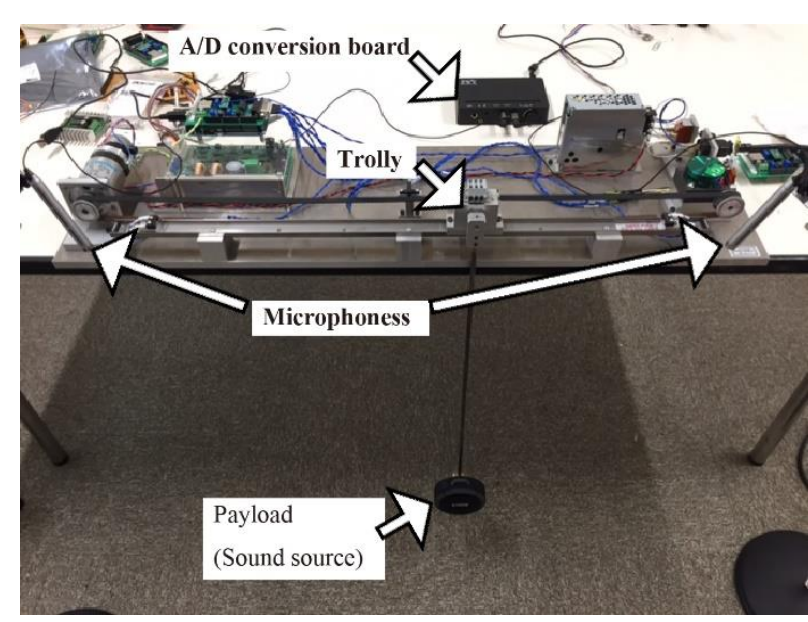

Fig. 2. Experimental equipment

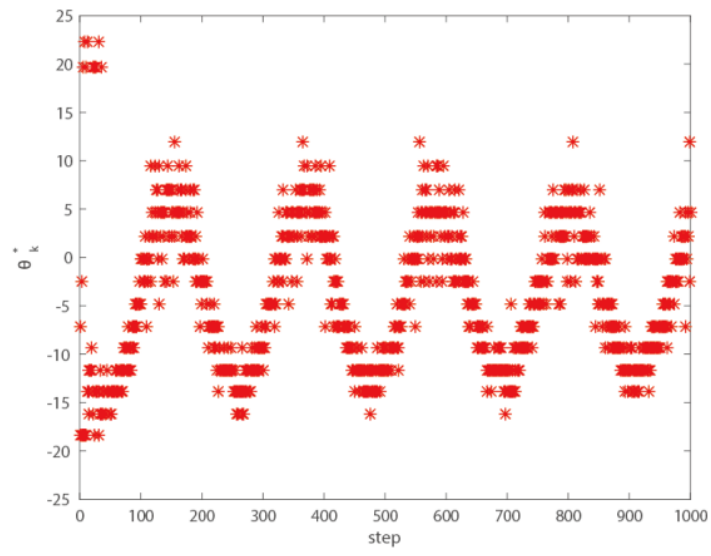

Fig. 3. Experimental result with $x=20$

where $\theta_{n}$ is the angle at $n$-th iteration. 


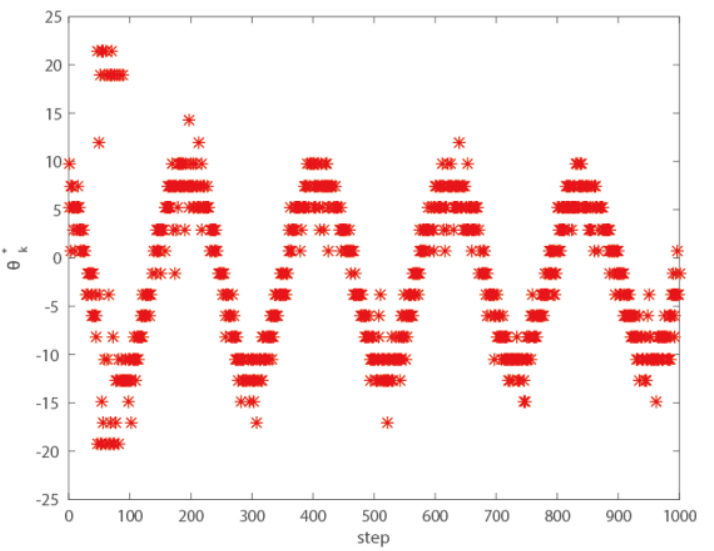

Fig. 4. Experimental result with $x=30$

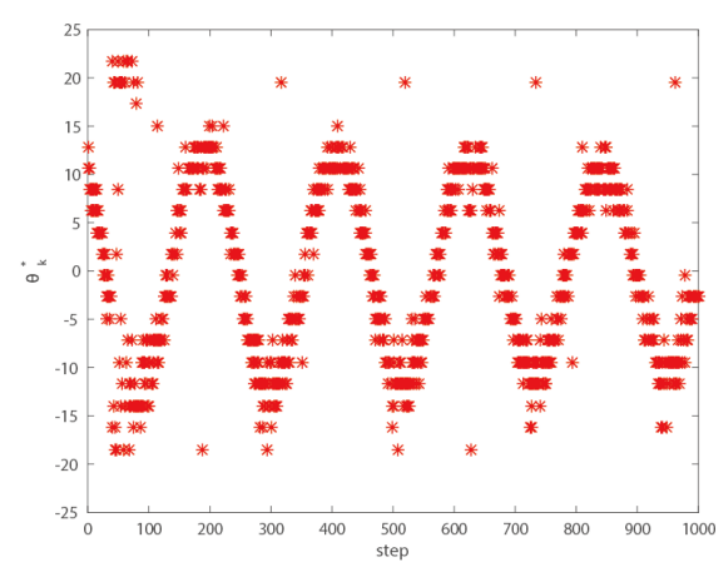

Fig. 5. Experimental result with $x=40$

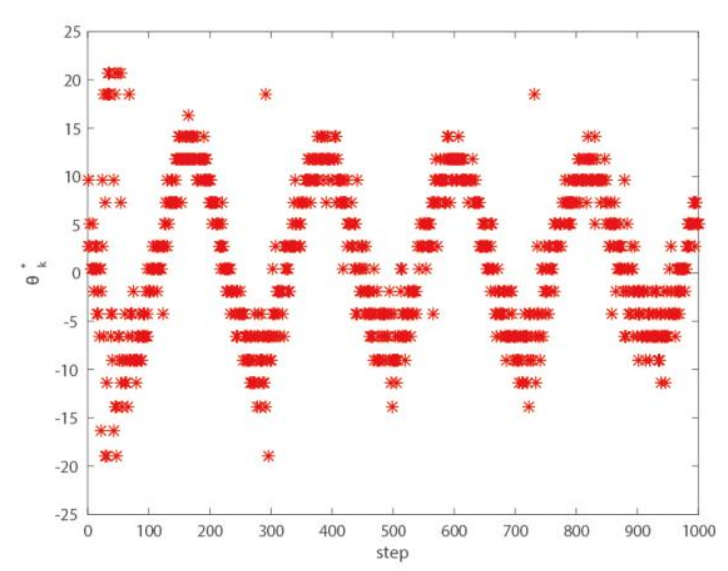

Fig. 6. Experimental result with $x=50$

\section{Conclusion}

We have proposed the measurement method of sway angle for anti-sway control of overhead crane with two microphones. The method uses a time delay of arrival (TDOA) of acoustic signals measured from the microphones. Also, we have presented the algorithm to obtain the sway angle from the TDOA with the Newton's method. In experimental results, we demonstrate that our sound-based measurement method can follow the sway angle when the payload is moving.

\section{References}

1. C.-Y Chang and H. W. Lie: Real-Time Visual Tracking and Measurement to Control Fast Dynamics of Overhead Cranes, IEEE Trans. Ind. Electron., vol.59, no. 3, pp.1640-1649, March 2012.

2. N. Sun and Y. Fang: New Energy Analytical Results for the Regulation of Underactuated Overhead Cranes: An End-Effector Motion-Based Approach, IEEE Trans. Ind. Electron., vol59, no.12, pp.47234734, Dec. 2012

3. L.-H. Lee. C.-H. Huang, S.-C. Ku, Z.-H. Yang, C.Y. Chang: Efficient Visual Feedback Method to Control a Three-Dimensional Overhead Crane, IEEE Trans. Ind. Electron., vol61, no.8, pp.40734083, Aug.2014.

4. K. Zavari, G. Pipeleers and J. Swevers: GainScheduled Controller Design: Illustration on an Overhead Crane, IEEE Trans. Ind. Electron., vol.61, no.7, pp.3713-3718, July 2014.

5. N. Sun, Y. Fang and H. Chen: A New Antiswing Control Method for Underactuated Cranes With Unmodeled Uncertainties: Theoretical Design and Hardware Experiments, IEEE Trans. Ind. Electron., vol. 59, no.3, pp.453-465, Jan. 2015.

6. M. Matsunaga, M. Nakamoto and T. Yamamoto: An acoustic signal processing for anti-sway control of overhead crane system, Proc. of 2017 International Workshop on Smart Info-Media Systems in Asia (SISA 2017), pp.124-127, Sept. 2017. 